\title{
Albumin permeability in isolated glomeruli in incipient experimental diabetes mellitus
}

\author{
M. Carraro, W. Mancini, M.Artero, C.Zennaro, L.Faccini, R. Candido, L. Armini, M. Calci, R. Carretta, B. Fabris \\ Institute of Clinical Medicine, University of Trieste, Trieste, Italy
}

\section{Abstract}

Aims/hypothesis. The pre-clinical phase of diabetic nephropathy is characterised by increased glomerular filtration rate and episodes of microalbuminuria. The cause of the microalbuminuria has been variably ascribed to alterations of the size or charge selective barriers of the glomerulus or both or as a consequence of the haemodynamic changes. Our aim was to investigate very early albumin permeability alterations in isolated glomeruli which were not subject to perfusion pressure.

Methods. Isolated glomeruli were studied from 120 male Wistar rats, divided into three groups: streptozotocin-treated, streptozotocin-treated with insulin pellet implants, and controls. From each group ten animals were killed at 7,14, 28, and 56 days after induction. Study variables included blood pressure, proteinuria, iopamidol clearance, albumin permeability and glomerular area. Subsequently, albumin permeability, proteinuria, and iopamidol clearance were determined in an additional group of 40 diabetic animals studied at $24,72,96$, and $120 \mathrm{~h}$ after induction.

Results. Albumin permeability increased steadily from induction in streptozotocin-treated animals, reaching a plateau at approximately $120 \mathrm{~h}$. Glomerular filtration rate was shown to increase significantly at approximately 7 days and proteinuria correlated with it. Glomerular hypertrophy was observed both in streptozotocin-treated animals and in streptozotocin-treated rats with insulin pellet implants. Strict blood glucose control delayed the appearance of the permeability defect in isolated glomeruli and inhibited the increase in glomerular filtration in intact animals. It did not prevent glomerular hypertrophy.

Conclusion/interpretation. An albumin permeability defect exists early in isolated non-perfused glomeruli from streptozotocin-treated rats and seems to be independent of glomerular filtration rate alterations. [Diabetologia (2000) 43: 235-241]

Keywords Diabetic nephropathy, albumin permeability, streptozotocin, blood glucose, proteinuria.
The transition from normal albumin permeability to microalbuminuria represents a critical juncture in the course of both insulin-dependent and non-insulin-dependent diabetes mellitus, in that it presages

Received: 17 August 1999 and in revised form: 11 November 1999

Corresponding author: M. Carraro, MD, Istituto di Medicina Clinica, Università degli Studi di Trieste, Ospedale di Cattinara, Strada di Fiume, 447, I-34149 Trieste, Italy

Abbreviations: $\mathrm{P}_{\mathrm{alb}}$, Albumin permeability value; GFR, glomerular filtration rate. not only diabetic nephropathy [1] but cardiovascular disease and death as well $[2,3]$. The pathophysiology underlying microalbuminuria is not well understood, but it is clear that both glomerular filtration rate and urinary albumin excretion are increased in newly diagnosed diabetic patients with poor glycaemic control. Altered size- or charge-selective characteristics of the glomerular capillary wall or both could explain the enhanced albumin permeability leading to microalbuminuria. Not all investigators have, however, detected an early size-selective defect using renal clearances of neutral dextrans [4-7] and the often transient nature of the microalbuminuria in early diabe- 
tes argues against a fixed reduction of negative charges in the permeability barrier. Another explanation involves the close correlation between the increased filtration fraction observed in microalbuminuric Type I (insulin-dependent) diabetes mellitus patients compared with controls and urinary albumin excretion [8]. Thus, reversible haemodynamic factors could play an important part in the pathogenesis of microalbuminuria [9].

Few published studies have focused on the events immediately following the induction of diabetes in experimental animals. Given the uncertainties regarding the origin of the altered glomerular albumin permeability in Type I diabetes, we were interested in determining firstly if a permeability defect in incipient diabetic nephropathy could be detected in the absence of haemodynamic forces (i.e., in isolated glomeruli), secondly the temporal relation between the alterations in glomerular filtration and the appearance of the permeability defect after the onset of hyperglycaemia and thirdly the effect of strict metabolic control on the course of the permeability defect after the onset of hyperglycaemia. The study design used oncotic gradients applied to isolated glomeruli to calculate an albumin permeability value $\left(\mathrm{P}_{\mathrm{alb}}\right)$, based on the previously developed technique $[10,11]$. This technique eliminates perfusion pressure as a variable in the determination of permeability.

\section{Methods}

\section{Experimental animals and study protocol}

Longitudinal albumin permeability study. Glomeruli were isolated from 80 male Wistar-Kyoto rats (age 2 months, weight 200-220 g), obtained from the colony maintained at the University of Trieste. They were separated into two equal groups. Group 1 animals were injected through the femoral vein with streptozotocin $55 \mathrm{mg} / \mathrm{kg}$ to induce diabetes mellitus; during the subsequent observation period the animals were maintained with ultralente insulin in minimal doses to prevent hyperglycaemic coma and death. The remaining 40 rats served as controls (Group 3). All animals were housed in the animal facility and fed standard rat chow without water restriction, according to the humane treatment guidelines established by the University.

From each group ten animals were killed at 7, 14, 28, and 56 days after induction of diabetes. Study variables included serum glucose and creatinine which were measured by autoanalyser, kidney and body weight, water intake and urine output measured in metabolic cages the day before death, proteinuria which was measured by colorimetry using a kit (Bio-Rad Protein Assay, Bio-Rad Laboratories, Milan, Italy) and glycosuria which was measured by autoanalyser. Blood pressure was measured by tail sphygmomanometry (Model 8005, W + W Electronic Scientific Instruments, Basel, Switzerland); the animals had been conditioned to the apparatus before the start of the experiment. Glomerular filtration rate (GFR) was approximated in our laboratory by a single injection technique measuring iopamidol clearance per $100 \mathrm{~g}$ body weight in a single plasma sample [12]. Iodine concentration in plasma was analysed by X-ray fluorescence (Renalyzer PRX 90, Provalid, Stockholm, Sweden) [13]. Iopamidol was injected through the tail vein and the blood sample drawn at $43 \mathrm{~min}$. This method was validated in diabetic animals by comparing it with a constant infusion method using ${ }^{51}$ Chromium-EDTA for measurement of GFR $(r=0.91$, slope $=0.93, p<0.001, n=12)$.

Hyperglycaemia was also induced with streptozotocin in a separate group of 40 animals and albumin permeability (described below), proteinuria and iopamidol clearance were determined at 24, 72, 96 and $120 \mathrm{~h}$ after induction.

Strict glucose control study. Concomitantly with the albumin permeability study, a second group of 40 rats (Group 2) was treated with streptozotocin, followed $48 \mathrm{~h}$ later by subcutaneous implantation of a sustained release insulin pellet (Linplant, LinShin Canada, Scarborough, Ontario, Canada). The pellet $(7 \times 2 \mathrm{~mm})$, which is available only for animal research purposes, releases regular insulin at a constant rate of 2 units $/ 24 \mathrm{~h}$ for 40 days, after which a new pellet must be implanted. The streptozotocin injections and implantation of the insulin pellets were done in all animals under ether anaesthesia. The study variables described above were also determined under conditions of strict glucose control at 7, 14, 28 and 56 days after induction of diabetes. The target of normoglycaemia in Group 2 rats was controlled by determining the glycosuria.

\section{Isolation of glomeruli and measurement of $P_{\text {alb }}$}

The animals were anaesthetised with ether, decapitated and the kidneys excised. Glomeruli were isolated from three-quarters of the renal cortex by standard sieving techniques in medium containing, in $\mathrm{mmol} / \mathrm{l}$ : sodium chloride 115, potassium chloride 5 , sodium acetate 10 , dibasic sodium phosphate 1.2 , sodium bicarbonate 25 , magnesium sulphate 1.2 , calcium chloride 1.0 and glucose 5.5. The $\mathrm{pH}$ had been titrated to 7.4. The medium also contained BSA $50 \mathrm{~g} / \mathrm{l}$ as an oncotic agent. The isolated glomeruli, which were free from capsule and arterioles, were then washed in one $\mathrm{ml}$ fresh medium and an aliquot of $0.1 \mathrm{ml}$ was incubated in $0.9 \mathrm{ml}$ of medium for $10 \mathrm{~min}$ at $37^{\circ} \mathrm{C}$. The glomeruli were then transferred to a glass cover slip coated with poly-L-lysine as an adherent and covered with fresh medium.

The rationale and methodology for the determination of albumin permeability has been described in detail $[10,11]$. In brief, each of 10 to 16 glomeruli per animal were videotaped through an inverted microscope before and after a medium exchange to one containing BSA $10 \mathrm{~g} / \mathrm{l}$. The medium exchange created an oncotic gradient across the basement membrane resulting in a glomerular volume change $\left(\Delta \mathrm{V}=\left(\mathrm{V}_{\text {final }}-\mathrm{V}_{\text {initial }}\right) /\right.$ $\left.\mathrm{V}_{\text {initial }}\right)$ which was measured off-line by a video-based image analysis program (MCID, Imaging Research, St. Catharines, Ontario, Canada). The computer program determines the average radius of the glomerulus in two-dimensional space and the volume is derived from the formula $V=4 / 3 \pi r^{3}$. The magnitude of $\Delta \mathrm{V}$ was related to the albumin reflection coefficient, $\sigma_{\mathrm{alb}}$, by the following equation: $\left(\sigma_{\mathrm{alb}}\right)_{\text {experimental }}=(\Delta \mathrm{V})_{\text {experimen- }}$ tal $/(\Delta \mathrm{V})_{\text {control }}$; the $\sigma_{\text {alb }}$ of the control glomeruli was assumed to be equal to 1 . The $\mathrm{P}_{\mathrm{alb}}$ is defined as $\left(1-\sigma_{\mathrm{alb}}\right)$ and describes the movement of albumin subsequent to water flux. When $\sigma_{\text {alb }}$ is zero, albumin moves across the membrane with the same velocity as water and $\mathrm{P}_{\mathrm{alb}}$ is 1.0. Conversely, when $\sigma_{\mathrm{alb}}$ is 1.0 , albumin cannot cross the membrane with water and $\mathrm{P}_{\mathrm{alb}}$ is zero.

Parenthetically, these assumptions are valid only when the response to an impermeable solute, such as high molecular 

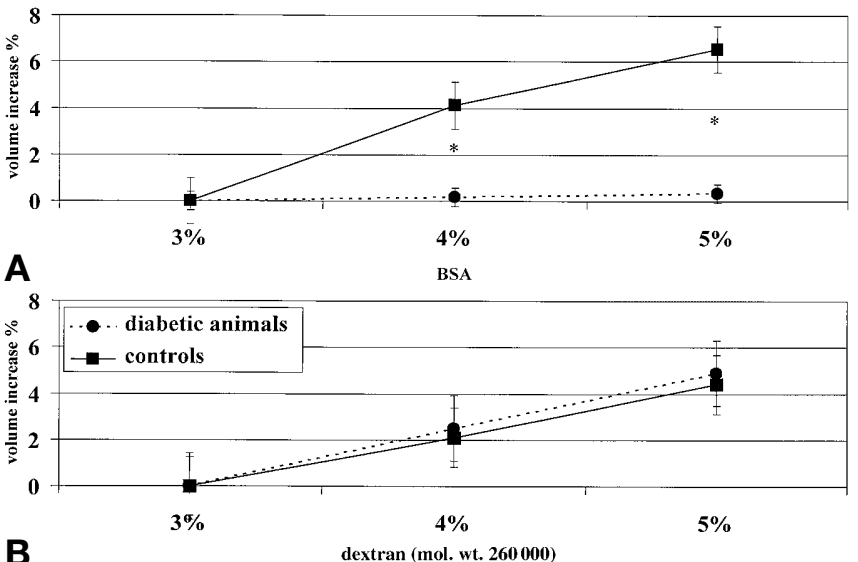

B

dextran (mol. wt. 260000 )

Fig. 1A, B. Volume responses of isolated glomeruli exposed to oncotic gradients induced by various concentrations of BSA (A) or high molecular weight dextran (B) from control and diabetic rats 14 days after induction of hyperglycaemia. $* p<0.01$. - - - diabetic animals, - - - controls

weight neutral dextran, is not altered by the experimental manipulations. We did these proofs at concentrations of $3 \%, 4 \%$ and $5 \%$ for both BSA and dextran (mol.wt. 260000$)$ in control and diabetic animals at 14 days (Fig. 1 ) and the volume response was not altered to dextran but was considerably diminished to albumin. The experimental manipulations were also done in medium containing high glucose concentrations (11.1 and $22.2 \mathrm{mmol} / \mathrm{l}$ ), the presence of which did not alter the normal glomerular behaviour to the oncotic gradient (mean $\mathrm{P}_{\mathrm{alb}}=0.19$ with glucose $22.2 \mathrm{mmol} / \mathrm{l}$ in both the incubation medium and the exchange medium; mean $\mathrm{P}_{\mathrm{alb}}=0$ with glucose $22.2 \mathrm{mmol} / \mathrm{l}$ in only the incubation medium).

\section{Morphometric analysis}

The remaining half kidney from Group 1, Group 2 and control animals was fixed in neutral formalin, processed through graded ethanol solutions, cleared in xylol and embedded in paraffin. For light microscopy study two random sections of $2 \mu \mathrm{m}$ mean thickness from each kidney were stained with haematoxylin and eosin. Each section was placed in a microscope and recorded by a colour videocamera; the image was then transferred by computer to the same video-based image analysis system as was used for measurement of $\mathrm{P}_{\mathrm{alb}}$. The area of 100 consecutive glomerular tufts sectioned at the vascular pole were measured in triplicate by two independent observers. Interobserver variability between measurements for all sections was less than $5 \%$. The reported values of the glomerular area represent the mean of the measurements obtained by both examiners.

\section{Statistical analysis}

The data are described as means \pm standard error of the mean. Comparisons among groups throughout the study period were done by ANOVA and subsequent Scheffe $F$ test. Variables within one group at different times were compared by ANOVA for repeated measures. Student's $t$ test for unpaired samples was used to compare control and diabetic animals in the longitudinal study. Linear regression analysis tested two-variable relations. Stat View 512 software (SAS Institute, Cary, N.C., USA) for an Apple Macintosh computer was used for the analysis.

In human studies, in which oncotic gradients applied to isolated rat glomeruli incubated in the presence of human serum have been used to predict the recurrence of focal segmental glomerular sclerosis after renal transplantation [14], a value of 0.50 has been used above which $\mathrm{P}_{\mathrm{alb}}$ is considered significant. In our study, because the glomeruli were not treated in vitro and the characteristics of the animals could be strictly controlled, we elected to use $\mathrm{P}_{\mathrm{alb}}$ as a continuous variable.

\section{Results}

Blood glucose was greatly increased in the diabetic rats, as expected (Table 1). Renal weight, water intake, and urine output were also significantly increased in the diabetic rats, whereas body weight was consistently decreased. Blood pressure tended to be higher in the diabetics, although it did not differ statistically significantly among groups at any point during the observation period. The insulin pellet provided very tight control of blood glucose, at times (day 14) in the hypoglycaemic range. There were no significant differences between Group 2 and control rats in glycosuria at any time, indicating that target blood glucose control had been achieved.

Iopamidol clearance as an estimate of GFR was significantly increased in relation to controls beginning at day 7 (Fig.2). Rats treated with insulin did not differ significantly from controls. The pattern of protein excretion tended to follow that of GFR for all groups (Fig. $2 ; r=0.49, p<0.0001$ ). Serum creatinine was also increased in the diabetic group. Because iothalamate clearance was also high, the increased creatinine could reflect an increase in either muscle catabolism or nitrogen intake.

Albumin permeability increased rapidly and remained high during the entire observation period in the diabetic rats (Fig. 2). Once this rapid increase of $\mathrm{P}_{\mathrm{alb}}$ was observed at seven days, we induced hyperglycaemia with streptozotocin in another group of 40 animals as described in Methods and measured albumin permeability at 24, 72, 96 and $120 \mathrm{~h}$ after induction. There was a steady increase in $\mathrm{P}_{\mathrm{alb}}$ $\left(\mathrm{P}_{\mathrm{alb}}\right.$ equal to $0.12,0.20,0.32,0.52$ at $24 \mathrm{~h}, 72 \mathrm{~h}$, $96 \mathrm{~h}$, and $120 \mathrm{~h}$, respectively). Iothalamate clearance increased in tandem with the observed permeability values in vitro $(1.041,1.200,1.246,1.238 / 100 \mathrm{~g} \mathrm{BW}$ at $24 \mathrm{~h}, 72 \mathrm{~h}, 96 \mathrm{~h}$, and $120 \mathrm{~h}$, respectively; $r=0.39$, $p<0.005$ ); no value before 5 days was, however, significantly greater than the control. The $\mathrm{P}_{\mathrm{alb}}$ of rats treated with insulin also increased slightly at 28 and 56 days compared with controls. The correlation between proteinuria and $\mathrm{P}_{\mathrm{alb}}(r=0.35$, $p=0.034$ ) was less close than that between proteinuria and GFR. 

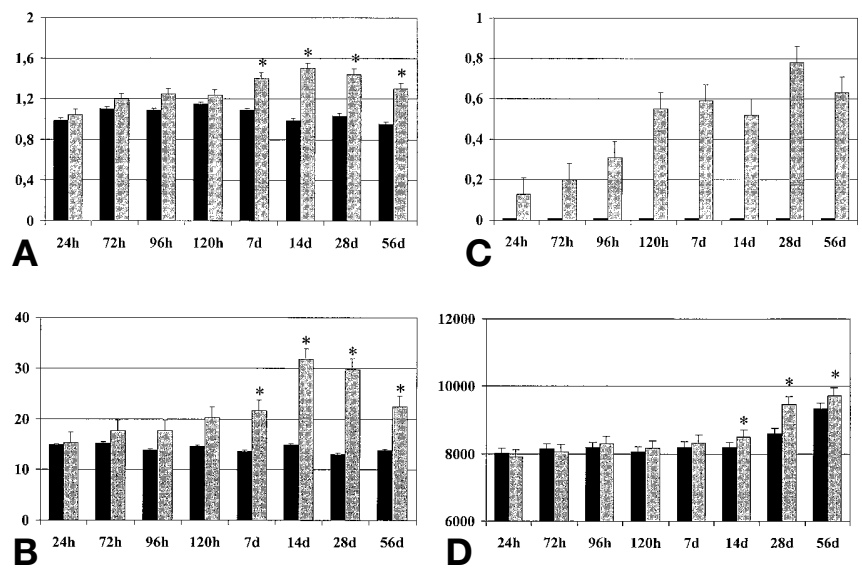

Fig. 2 A-D. Histograms representing the data (means \pm SEM) from diabetic (grey bars) and control (black bars) animals during the longitudinal study. ${ }^{*} p<0.01$, compared with controls. A Iopamidol clearance as an estimate of GFR $\left(\mathrm{ml} \cdot \mathrm{min}^{-1}\right.$. $100 \mathrm{~g}^{-1}$ body weight) between groups. B Protein excretion (mg/day) between groups. C Albumin permeability $\left(\mathrm{P}_{\mathrm{alb}}\right)$ determined in isolated glomeruli exposed to oncotic gradients during the course of the experiment. D Glomerular area $\left(\mu \mathrm{m}^{2}\right)$ between groups during the observation period. $d=$ days

The results of the ANOVA for repeated measures within the diabetic group show that GFR and proteinuria at 7 days were significantly higher than the values obtained before 7 days and subsequently did not vary among time periods whereas behaviour of $\mathrm{P}_{\mathrm{alb}}$ was the same beginning at $120 \mathrm{~h}$.

Glomerular hypertrophy was first noted in the animals treated with streptozotocin at 14 days (Fig. 2). Because these young rats were growing, the area of control glomeruli also increased during the observation period but the areas of the diabetic glomeruli were significantly greater than controls at 14 and 28 days, as were those from the animals treated with insulin (Fig. 3).

\section{Discussion}

Reversible haemodynamic factors are thought to play a prominent part in the pathogenesis of microalbuminuria in Type I diabetes. For example, diabetic patients have a more conspicuous increase of proteinuria following moderate exercise $[15,16]$. This phenomenon is thought to be due to an increased filtration fraction, providing a driving force for albumin infusion into Bowman's space. Poor metabolic control, could provide a similar haemodynamic stimulus for albumin leakage perhaps by enhanced angiotensin II secretion. Of the four determinants of glomerular filtration (plasma flow, systemic oncotic pressure, transcapillary pressure, and the ultrafiltration coefficient, which is the product of capillary hydraulic conductivity and capillary surface available for filtration) plasma flow $[17,18]$, transcapillary pressure [17] and cap- 

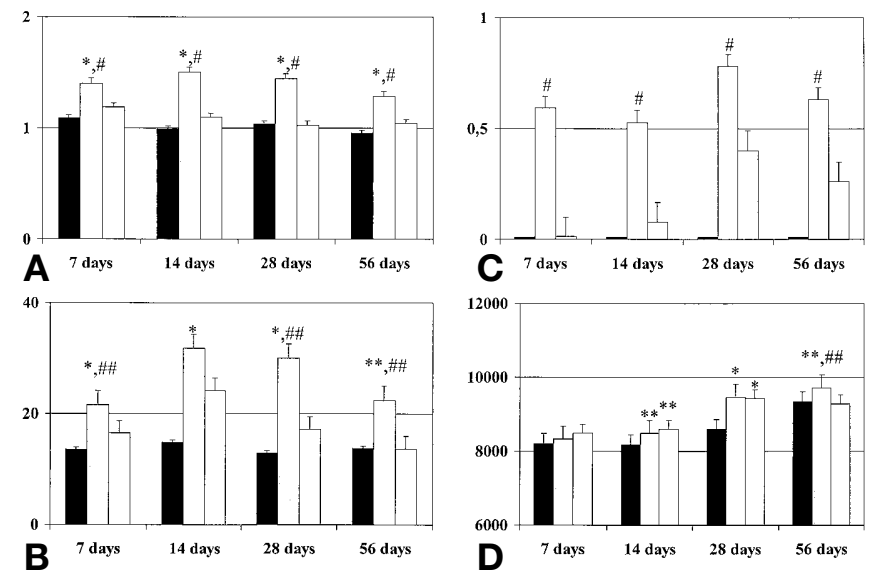

Fig.3 A-D. Histograms representing the data (means \pm SEM) in the strict glucose control study animals. Black bars represent control animals, grey bars diabetic animals and white bars diabetic animals implanted with the insulin pellet. $* p<0.01$, vs controls; ${ }^{* *} p<0.05$, vs controls; ${ }^{\#} p<0.01$, vs insulin pellet; $\# p<0.05$, vs insulin pellet. A Iopamidol clearance as an estimate of GFR $\left(\mathrm{ml} \cdot \mathrm{min}^{-1} \cdot 100 \mathrm{~g}^{-1}\right.$ body weight $)$ between groups. B Protein excretion ( $\mathrm{mg} /$ day) between groups. C Albumin permeability $\left(\mathrm{P}_{\mathrm{alb}}\right)$ determined in isolated glomeruli exposed to oncotic gradients during the course of the experiment. D Glomerular area $\left(\mu \mathrm{m}^{2}\right)$ between groups during the observation period.

illary surface area $[19,20]$ are all increased in early diabetes. Systemic oncotic pressure seems to be unchanged in Type I diabetes, and in isolated glomeruli it has been shown that hydraulic conductivity could be reduced in early diabetes [21].

Despite the cogent arguments for a haemodynamic basis of diabetic microalbuminuria, our study shows that an albumin permeability defect exists in the earliest stages of Type I diabetes induced by streptozotocin, in the absence of perfusion in isolated rat glomeruli. The rapidity with which the defect appears is consistent with recent studies which have shown rapid mRNA up-regulation of a number of cytokines, growth factors and extracellular matrix proteins in glomerular cells cultured in the presence of high glucose concentrations within $24-48 \mathrm{~h}$, which could result in damage in vivo to the permeability barrier. These auto-factors and paracrine factors include TGF- $\beta$ [22], IGF-1, the beta chain of platelet derived growth factor and fibroblast growth factor [23] as well as renin and angiotensin II receptor type 1 receptors associated with increased nitric oxide synthetase [24]. Transcripts for fibronectin, laminin and type IV collagen are also increased, probably through activation of protein kinase $\mathrm{C}$ $[25,26]$. On the other hand, the up-regulation of fibronectin and collagen mRNA required 5 days of high glucose exposure in cultured endothelial cells in one study [27] and these investigators postulated that non-enzymatic glycation influenced intracellular signalling pathways, although it is unlikely that glycation plays an early part in structural alterations.

In another study [28] the fractional clearance of low molecular weight dextrans decreased in diabetic patients while the fractional clearance of dextrans with a high molecular weight increased. The finding that fractional clearance of dextrans and albumin increases even in early diabetes suggests that structural changes in the glomerular basement membrane, and not merely haemodynamic changes, are responsible for altered permselectivity [29]. Other studies [30, 31] show the proportion of the basement membrane occupied by small selective pores is decreased and the area containing large unselective pores is increased, allowing the passage of medium sized molecules such as albumin. Growth factors or extracellular matrix proteins could be involved in the modulation of pore configuration. Evidence of reduced glomerular size-selectivity and charge-selectivity has also been found in human non-diabetic subjects with microalbuminuria [32] and these investigators also proposed a structural alteration of the glomerular filtration barrier. Finally, the often evanescent nature of microalbuminuria in incipient diabetes could result from hormonal alteration of the capillary surface available for passage of larger molecules such as albumin. For example, angiotensin II could influence glomerular size permselectivity of neutral dextrans, a phenomenon prevented by infusion of angiotensin II inhibitors [33].

Our finding that $\mathrm{P}_{\mathrm{alb}}$ also slightly increases eventually in the rats treated with insulin agrees with recent observations that in Type II (non-insulin-dependent) diabetes mellitus, hyperinsulinaemia increases urinary albumin excretion, as well as the proximal tubular enzyme $N$-acetyl- $\beta$-D-glucosaminidase but not the distal Tamm-Horsfall protein, by unknown mechanisms [34].

In previous studies of albumin permeability associated with diabetes $[6,30,31,35,36]$, the relation between urine and plasma concentration (fractional clearance) of neutral or charged dextrans provided the basis for the study of variations of the permeability barrier. The fractional clearance of mid-sized proteins such as albumin increases, however, not only when there is a permeability change of the capillary wall but also when the GFR is diminished, such as after angiotensin II infusion [37]. Thus, it could be difficult to distinguish the individual contributions of haemodynamic factors or structural alterations of the capillary wall or both to increased albumin permeability. Pressure and flow variables are avoided in the in vitro determination of $\mathrm{P}_{\mathrm{alb}}$ in isolated glomeruli, which we used in this study. Oncotic gradients applied to isolated glomeruli, in the absence of perfusion or an immunological response, have also been used to determine the effects of single mediators such as superoxide [38], the terminal components of 
complement and the Heymann antibody [39] and others, which could be involved in glomerular injury.

Our study recapitulated many previous experiments which showed increased GFR in the incipient stages of diabetic nephropathy. Note, however, that even at the earliest time points, GFR alterations were accompanied by in vitro evidence of a permeability defect. In other words, glomerular hyperfiltration did not seem to precede the in vitro albumin permeability defect. The time courses for statistically significant alterations of the permeability defect and GFR do not coincide. The longitudinal study suggested that $\mathrm{P}_{\mathrm{alb}}$ increases steadily from induction and seems to plateau at approximately $120 \mathrm{~h}$, whereas GFR increased in a stepwise manner at approximately 7 days. The pattern of proteinuria tended to follow that of the GFR. Thus, it could be hypothesised that increased GFR promotes microalbuminuria in the incipient stages of diabetic nephropathy in the presence of a structural permeability defect.

As in human subjects [40], strict metabolic control abrogated the increased GFR, the proteinuria and statistically significantly delayed the appearance of the permeability defect. That the insulin pellet was implanted at $48 \mathrm{~h}$ and a permeability alteration was noted at $24 \mathrm{~h}$, in the absence of tight control, could be interpreted not only as a prevention but also perhaps as a reversal of the intracellular processes which eventually give rise to the permeability defect.

Glomerular hypertrophy in diabetic animals compared with controls was first seen at 14 days. That the animals treated with insulin also had enlarged glomeruli is consistent with other findings [41]; although renal hypertrophy was normalised with good metabolic control, mesangial expansion persists with insulin treatment. In contrast, good blood glucose control has been found to prevent mesangial expansion [42]. The same investigator reported recently, however, only a partial reduction of glomerular volume fractions after insulin treatment in diabetic animals [43]. The interpretation of these conflicting results is complicated by the considerable variation in studies between duration of diabetes, modality of insulin treatment, type of insulin and methods of glomerular enlargement estimation. In our study tight glucose control prevented increased GFR and microalbuminuria but did not prevent glomerular hypertrophy, perhaps as a result of the growth promoting potential of insulin. It is possible that insulin in vivo might activate glomerular growth either directly or through pathways involving other growth factors and cytokines, such as TGF- $\beta 1$ or activation of the insulin-like growth factor-1 receptor. Long-term insulin exposure also increases renal angiotensin II receptor density [44], thus facilitating the growth promoting action of angiotensin II.

A permeability defect exists in the absence of glomerular perfusion very early in the course of incipient diabetic nephropathy in rats treated with streptozotocin. This defect seems to arise independently of GFR, which was found to be significantly greater than controls at 7 days. Proteinuria was highly correlated with GFR. Strict blood glucose control delays (or reverses) the appearance of the permeability defect and inhibits the increase in GFR and proteinuria. Tight control does not prevent glomerular hypertrophy.

Acknowledgements. This work was presented in part as an oral communication at the XXXV Congress of the European Renal Association - European Dialysis and Transplant Association, Rimini (Italy), June 6-9, 1998. We thank Drs. B. Biasioli and G. Cortelli, Division of Clinical Pathology, Ospedale Maggiore, Trieste, for laboratory assistance.

\section{References}

1. Viberti GC, Jarrett RJ, Mahmud U, Hill RD, Argyropoulos A, Keen H (1982) Microalbuminuria as a predictor of clinical nephropathy in insulin-dependent diabetes mellitus. Lancet i: $1430-1432$

2. Yudkin JS, Forrest RD, Jackson CA (1988) Microalbuminuria as a predictor of vascular disease in non-diabetic subjects. Lancet ii: $530-533$

3. Damsgaard EM, Froland A, Jorgensen OD, Mogensen CE (1990) Microalbuminuria as predictor of increased mortality in elderly people BMJ 300: 297-308

4. Mogensen CE (1971) Kidney function and glomerular permeability to macromolecules in early juvenile diabetes Scand J Clin Lab Invest 28: 79-90

5. Pennell JP, Yanagawa N, Hwang KH, Millard MM, Bourgoignie JJ (1981) Glomerular selective permeability to macromolecular neutral dextrans in experimental diabetes Diabetologia 20: 223-227

6. Michels LD, Davidman M, Keane WF (1982) Glomerular permeability to neutral and anionic dextrans in experimental diabetes. Kidney Int 21: 699-705

7. Deckert T, Kofoed-Enevoldsen A, Vidal P, Norgaard K, Andreasen HB, Feldt-Rasmussen B (1993) Size- and charge selectivity of glomerular filtration in Type I (insulin-dependent) diabetic patients with and without albuminuria. Diabetologia 36: 244-251

8. Feldt-Rasmussen B (1989) Microalbuminuria and clinical nephropathy in type 1 (insulin-dependent) diabetes mellitus: pathophysiological mechanisms and intervention studies. Dan Med Bull 36: 405-415

9. Hommel E, Mathiesen E, Edsberg B, Bahnsen M, Parving $\mathrm{H}-\mathrm{H}$ (1986) Acute reduction of arterial blood pressure reduces urinary albumin excretion in Type I (insulin-dependent) diabetic patients with incipient nephropathy. Diabetologia 29: 211-215

10. Savin VJ, Terreros DA (1981) Filtration in single isolated mammalian glomeruli. Kidney Int 20: 188-197

11. Savin VJ, Sharma R, Lovell HB, Welling DJ (1992) Measurement of albumin reflection coefficient with isolated rat glomeruli. J Am Soc Nephrol 3: 1260-1269

12. Lundquist S, Leander P (1991) The extrarenal clearance of water-soluble iodinated contrast media: an experimental study in rabbits. Invest Radiol 26 [Suppl 1]:S75-S79

13. Boijsen M, Jacobsson L, Tylén U (1987) Renal function measured by x-ray fluorescence analysis: a comparison between contrast medium clearance and non-simultaneous 51Cr-EDTA clearance. Acta Radiol 28: 581-585 
14. Savin VJ, Sharma R, Sharma M et al. (1996) Circulating factor associated with increased glomerular permeability to albumin in recurrent focal segmental glomerulosclerosis. N Engl J Med 334: 878-883

15. Vittinghus E, Mogensen CE (1982) Graded exercise and protein excretion in diabetic man and the effect of insulin treatment. Kidney Int 21: 725-729

16. Feldt-Rasmussen B, Baker L, Deckert T (1985) Exercise as a provocative test in early renal disease in Type I (insulindependent) diabetes: albuminuric, systemic and renal haemodynamic responses. Diabetologia 28: 389-396

17. Hostetter TH, Troy JL, Brenner BM (1981) Glomerular hemodynamics in experimental diabetes mellitus. Kidney Int 19: 410-415

18. Sandahl Christiansen J, Gammelgaard J, Frandsen M, Parving H-H (1981) Increased kidney size, glomerular filtration rate and renal plasma flow in short-term insulin-dependent diabetics. Diabetologia 20: 451-456

19. Kroustrup JP, Gundersen HJG, Osterby R (1977) Glomerular size and structure in diabetes mellitus. III. Early enlargement of the capillary surface. Diabetologia 13: 207-210

20. Hirose K, Tsuchida H, Osterby R, Gundersen HJG (1980) A strong correlation between glomerular filtration rate and filtration surface in diabetic kidney hyperfunction. Lab Invest 43: 434-437

21. Ellis EN, Wiegmann TB, Savin VJ (1992) Diminished glomerular capillary hydraulic conductivity precedes morphologic changes in experimental diabetes mellitus in the rat. Diabetes 41: 1106-1112

22. Shankland SJ, Scholey JW, Ly H, Thai K (1994) Expression of transforming growth factor- $\beta 1$ during diabetic renal hypertrophy. Kidney Int 46: 430-442

23. Abboud HE (1997) Growth factors and diabetic nephropathy: an overview. Kidney Int 52[Suppl 60]: S3-S6

24. Choi KC, Kim NH, An MR, Kang DG, Kim SW, Lee J (1997) Alterations of intrarenal renin-angiotensin and nitric oxide systems in streptozotocin-induced diabetic rats. Kidney Int 52[Suppl 60]: S23-S27

25. Ayo SH, Radnik RA, Garoni J, Glass WF, Kreisberg JI (1990) High glucose causes an increase in extracellular matrix proteins in cultured mesangial cells. Am J Pathol 136: $1339-1348$

26. Kreisberg JI, Radnik RA, Ayo SH, Garoni J, Saikumar P (1994) High glucose elevates c-fos and c-jun transcripts and proteins in mesangial cell cultures. Kidney Int 46: 105-112

27. Cagliero E, Roth T, Roy S, Lorenzi M (1991) Characteristics and mechanisms of high-glucose-induced overexpression of basement membrane components in cultured human endothelial cells. Diabetes 40: 102-110

28. Deen WM, Bridges CR, Brenner BM, Myers BC (1985) Heteroporous model of glomerular size selectivity: application to normal and nephrotic humans. Am J Physiol 249: F374-F389
29. Noth RH, Krolewski AS, Kaysen GA, Meyer TW, Schambelan M (1989) Diabetic nephropathy: hemodynamic basis and implications for disease management. Ann Intern Med 110: 795-813

30. Tomlanovich S, Deen WM, Jones HW, Schwartz HC, Myers BD (1987) Functional nature of glomerular injury in progressive diabetic glomerulopathy. Diabetes 36: 556-565

31. Friedman S, Jones HW, Golbetz HV, Lee JA, Little HL, Myers BD (1983) Mechanisms of proteinuria in diabetic nephropathy. II. A study of the size-selective glomerular filtration barrier. Diabetes 32[Suppl 2]: 40-46

32. Jensen JS, Borch-Johnsen K, Deckert T, Deckert M, Jensen G, Feldt-Rasmussen B (1995) Reduced glomerular size- and charge-selectivity in clinically healthy individuals with microalbuminuria. Eur J Clin Invest 25: 608-614

33. Yoshioka T, Mitarai T, Kon V, Deen WM, Rennke HG, Ichikawa I (1986) Role for angiotensin II in overt functional proteinuria. Kidney Int 30: 538-545

34. Catalano C, Muscelli E, Quinones Galvan A et al. (1997) Effect of insulin on systemic and renal handling of albumin in nondiabetic and NIDDM subjects. Diabetes 46: 868-875

35. Carrie BJ, Myers BD (1980) Proteinuria and functional characteristics of the glomerular barrier in diabetic nephropathy. Kidney Int 17: 669-676

36. Myers BD, Nelson RG, Tan M et al. (1995) Progression of overt nephropathy in non-insulin-dependent diabetes. Kidney Int 47: 1781-1789

37. Bohrer MP, Deen WM, Robertson CR, Brenner BM (1977) Mechanism of angiotensin II-induced proteinuria in the rat. Am J Physiol 233: F13-F21

38. Dileepan KN, Sharma R, Stechschulte D, Savin VJ (1993) Effect of superoxide exposure on albumin permeability of isolated rat glomeruli. J Lab Clin Med 121: 797-804

39. Savin VJ, Johnson RJ, Couser WG (1994) C5b-9 increases albumin permeability of isolated glomeruli in vitro. Kidney Int 46: 382-387

40. Wiseman MJ, Saunders AJ, Keen H, Viberti G (1985) Effect of blood glucose control on increased glomerular filtration rate and kidney size in insulin-dependent diabetes. $\mathrm{N}$ Engl J Med 312: 617-621

41. Stackhouse S, Miller PL, Park SK, Meyer TW (1990) Reversal of glomerular hyperfiltration and renal hypertrophy by blood glucose normalization in diabetic rats. Diabetes 39: 989-995

42. Rasch R (1979) Prevention of diabetic glomerulopathy in streptozotocin diabetic rats by insulin treatment. The mesangial regions. Diabetologia 17: 243-248

43. Rasch R, Dorup J (1997) Quantitative morphology of the rat kidney during diabetes mellitus and insulin treatment. Diabetologia 40: 802-809

44. Ling BN, Seal EE, Eaton DC (1993) Regulation of mesangial cell ion channels by insulin and angiotensin II. Possible role in diabetic glomerular hyperfiltration. J Clin Invest 92: 2141-2151 